\title{
Laser-induced manipulation of magnetic anisotropy and magnetization precession in an ultrathin cobalt wedge
}

\author{
J. Kisielewski, ${ }^{1,2, *}$ A. Kirilyuk, ${ }^{1}$ A. Stupakiewicz, ${ }^{2}$ A. Maziewski, ${ }^{2}$ A. Kimel, ${ }^{1}$ Th. Rasing, ${ }^{1}$ L. T. Baczewski, ${ }^{3}$ and A. Wawro ${ }^{3}$ \\ ${ }^{1}$ Radboud University Nijmegen, Institute for Molecules and Materials, Heyendaalseweg 135, 6525AJ Nijmegen, The Netherlands \\ ${ }^{2}$ Laboratory of Magnetism, University of Biatystok, Lipowa 41, 15-424 Biatystok, Poland \\ ${ }^{3}$ Institute of Physics, Polish Academy of Sciences, al. Lotników 32/46, 02-668 Warsaw, Poland \\ (Received 3 February 2012; revised manuscript received 20 April 2012; published 29 May 2012)
}

\begin{abstract}
Ultrafast magnetization dynamics in an epitaxially grown Co wedge-shaped layer sandwiched between $\mathrm{Pt}$ films has been studied by means of time-resolved magneto-optics. By changing either the external magnetic field or the thickness-dependent anisotropy field, we were able to tune the magnetization precession, as it depends on the effective magnetic field affecting the sample. A particularly interesting possibility of tuning occurs at the range of thicknesses near the spin reorientation transition (at $d_{0}=2.3 \mathrm{~nm}$ ), where the effective anisotropy field changes sign. To describe the measured dynamics we propose a model based on ultrafast thermal changes of the effective anisotropy field as a mechanism of triggering precession of the spins.
\end{abstract}

DOI: 10.1103/PhysRevB.85.184429

PACS number(s): 75.78.Jp, 75.30.Gw, 75.70.Ak

\section{INTRODUCTION}

Laser-induced magnetization dynamics is a fascinating area of research that uses femtosecond laser pulses to create the unique possibility of magnetization control on a subpicosecond time scale. ${ }^{1}$ This is orders of magnitude faster than using, for example, a magnetic field or current, the former approach being widely used in magnetic hard drives. ${ }^{2,3}$ This research also contributes to our fundamental understanding of the highly nonequilibrium processes involved in the interaction of spins with light at these short time scales. The participating mechanisms involve the energy and angular momentum transfer between photons, spins, charges, and the lattice. ${ }^{1}$ The situation is particularly complicated in metals, where the effects of heating occur at the time scale of the laser pulse duration. $^{4}$

There are different approaches to the manipulation of the magnetization direction using laser pulses, ranging from ultrafast demagnetization ${ }^{5}$ to nonthermal optomagnetic effects ${ }^{6}$ and all-optical switching. ${ }^{7}$ One of them is via the light-induced changes of the magnetic anisotropy, leading to a precessional motion of spins. This could eventually be used for switching, such as demonstrated in Refs. 8 and 9. This effect is most clearly demonstrated in dielectrics, ${ }^{10,11}$ where the anisotropy is induced via charge-transfer electronic transitions that in turn depend on the light polarization. In metals, on the contrary, the absorption of light is always accompanied by a redistribution of the energy over many degrees of freedom, resulting in a strong overall increase of temperature. As far as the magnetic anisotropy is considered, such an increase results in two effects: first, due to the thermal decrease of the magnetization, the demagnetizing field, or shape anisotropy, is reduced. And second, the magnetocrystalline anisotropy is temperature-dependent by itself, ${ }^{12,13}$ though even in statics this mechanism is still a subject of debates. ${ }^{14,15}$

Here we present our study of light-induced changes of the magnetic anisotropy in an ultrathin cobalt wedge-shaped film sandwiched between platinum layers. All-optically induced magnetic precession was used as a fingerprint of the ultrafast pulse-induced modification of the anisotropy. As in such wedged magnetic layer the static anisotropy changes continuously with the film thickness, ${ }^{16}$ it allowed us to clearly distinguish the contribution of the photo-induced anisotropy change to the excitation process. Varying the applied magnetic field helped us further to separate the different contributions. Even though the light-induced changes of magnetic anisotropy have been already discussed, ${ }^{9,17,18}$ there was no universal approach that could be applied to its description. In this paper we therefore study this process in detail, using a simple model that allows us to describe the experimental results and derive the temperature dependence of the interface anisotropy contribution. The fact that the anisotropy changes at the same time scale as the magnetization is found to be consistent with our data.

The paper is organized as follows: experimental details describing sample configuration and the magneto-optical techniques are given in Sec. II. In Sec. III we present the experimental results obtained from statics and dynamics measurements, and we discuss them on the basis of the proposed model.

\section{EXPERIMENT DETAILS}

The sample was produced by molecular beam epitaxy in a base pressure of $10^{-10}$ Torr. The following structure was grown on a sapphire single-crystal (11-20) wafer: (i) buffer layer of $20 \mathrm{~nm} \mathrm{Mo}(110)$ deposited at $T=1000^{\circ} \mathrm{C}$, (ii) 20-nm-thick $\mathrm{Pt}(111)$ underlayer deposited at room temperature, (iii) a $\operatorname{Co}(0001)$ wedge layer of $0-3 \mathrm{~nm}$ thickness range, produced with the shutter linearly moving during deposition, and (iv) an 8-nm-thick Pt overlayer to enhance the magneto-optical effects and prevent the Co film from oxidation. The evaporation sources were carefully calibrated and the thickness accuracy was better than 5\%. The growth of the sample was, whenever possible, monitored in situ by reflection high-energy electron diffraction (RHEED) and Auger spectroscopy. Two-dimensional growth mode of Pt and Co was confirmed by the presence of sharp streaks on RHEED patterns, resulting from a high quality of the sample. 
The static magnetization reversal process and magnetic anisotropy were studied by means of a magneto-optical Kerr effect (MOKE) magnetometer in polar (PMOKE) and longitudinal (LMOKE) configurations. The laser beam $(\lambda=$ $640 \mathrm{~nm}$ ) was focused on the Co wedge to a spot of $0.4 \mathrm{~mm}$ in diameter. The polar magnetization component was measured using PMOKE with close-to-normal incidence of the laser beam and the magnetic field applied perpendicularly to the sample plane.

The studies of ultrafast dynamics were carried out with a typical time-resolved pump-probe magneto-optical Kerr effect (TR-MOKE) setup. A Ti:sapphire oscillator (repetition rate $80 \mathrm{MHz}$, wavelength $800 \mathrm{~nm}$, pulse duration $80 \mathrm{fs}$ ) and regenerative amplifier (output repetition rate $250 \mathrm{kHz}$ ) were used. Laser pulses were split into pump and probe beams. The pump frequency was doubled with a nonlinear BBO crystal. The angles of incidence were $0^{\circ}$ for pump and $40^{\circ}$ for probe. The beams were focused at the sample surface to a spot diameter of 60 and $30 \mu \mathrm{m}$, for pump and probe, respectively, which was determined using a standard knife-edge method. ${ }^{19}$ Energy density of the pump beam was equal to $2.6 \pm$ $0.3) \mathrm{mJ} / \mathrm{cm}^{2}$. An external magnetic field was applied at the angle of $10^{\circ}$ to the sample plane, in the plane of light incidence. The Kerr rotation was measured with a split-diode detector and Wollastone prism, using a standard modulation technique with a chopper at the pump beam and lock-in amplifier. The Kerr rotation was proportional to the $\hat{z}$ component of the magnetization (perpendicular to the sample plane), as for that geometry the polar Kerr effect is much stronger than the longitudinal and transversal ones. ${ }^{20}$ All measurements were done at room temperature.

\section{RESULTS AND DISCUSSION}

\section{A. Thickness-driven spin-reorientation transition}

In an ultrathin Co film a strong perpendicular magnetic anisotropy results from a competition between magnetocrystalline volume and surface anisotropies, which is responsible for a spin reorientation transition (SRT) from perpendicular to an in-plane direction with increasing Co layer thickness. ${ }^{16,21}$ From the set of hysteresis curves measured in polar configuration (PMOKE) along the Co wedge we have determined a value of the SRT thickness to be equal to $d_{0}=2.3 \mathrm{~nm}$. Some examples of the hysteresis curves are displayed at the top of Fig. 1, for thicknesses both below and above the SRT. The thickness dependence of the normalized remanence (equivalent to the mean cosine of the magnetization orientation angle at zero external magnetic field), derived from the hysteresis loops, is shown as the black points in Fig. 1. Using the longitudinal magneto-optical Kerr effect (LMOKE) we have confirmed the absence of any preferred in-plane axis above the SRT, which means that the anisotropy is of an easy-plane type.

The uniaxial anisotropy energy for ultrathin Co films in hcp phases ${ }^{16}$ can be written as

$$
E_{\text {ani }}=K_{\text {eff }} \sin ^{2} \theta+K_{2} \sin ^{4} \theta+\ldots,
$$

where $\theta$ is the polar angle of the magnetization vector. The preferred direction of the magnetization is given by the minimum of the anisotropy energy. Taking into account

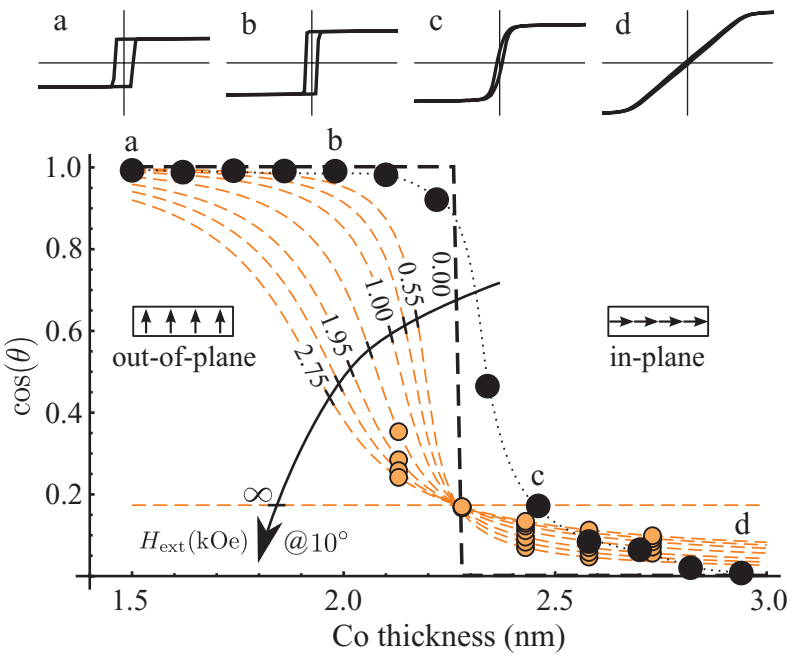

FIG. 1. (Color online) PMOKE hysteresis loops measured for selected Co layer thicknesses: (a) $1.5 \mathrm{~nm}$, (b) $2.0 \mathrm{~nm}$, (c) 2.5 $\mathrm{nm}$, and (d) $2.9 \mathrm{~nm}$. Cosines of static preferred magnetization orientation in the studied sample as a function of Co layer thickness: in zero-field (i.e., normalized remanence), measured (black points and dotted line as a guide to the eye) and calculated within the first anisotropy constant approximation (black dashed line). That dependence was also determined for the configuration of pump-probe measurements - in the presence of an external magnetic field applied at the angle of $10^{\circ}$ to the sample plane (orange dashed lines; field amplitude values: $0.55,0.75,1.00,1.45,1.95,2.35,2.75 \mathrm{kOe}$ and $\infty)$. Specified field amplitudes and Co thicknesses were used in the dynamics measurement (orange points).

the first order term only, there are two solutions, depending on the sign of $K_{1 \mathrm{eff}}$ : $\theta=0^{\circ}, 180^{\circ}$ (easy axis) for positive, and $\theta=90^{\circ}$ (easy plane) for negative $K_{1 \text { eff }}$. According to the phenomenological Néel formula, the effective anisotropy field $H_{1 \text { eff }}$, related to the first anisotropy constant, can be defined as a sum of bulk $\left(K_{v}\right)$ and surface $\left(K_{S}\right)$ contributions ${ }^{21,22}$ and a demagnetization term $(-M)$ :

$$
H_{1 \mathrm{eff}}=\frac{2}{\mu_{0} M}\left(K_{v}+\frac{2 K_{s}}{d}\right)-M .
$$

The effective anisotropy field depends on the layer thickness and can be either positive or negative. This results in the thickness-driven SRT at $d_{0}=2.3 \mathrm{~nm}$, where $H_{1 \text { eff }}$ crosses zero. In this first-order term approximation, the SRT is abrupt, as illustrated by the black dashed line of the calculated preferred magnetization orientation in Fig. 1. The clearly smoother measured normalized remanence dependence may indicate the necessity of involving the second order anisotropy constant $K_{2}$ too. This problem will be discussed later in Sec. III B.

The room-temperature value of $K_{v}=0.41 \mathrm{MJ} / \mathrm{m}^{3}$ for hcp bulk cobalt was taken from Refs. 16 and 23, while the surface anisotropy coefficient $K_{s}=(1.00 \pm 0.02) \mathrm{mJ} / \mathrm{m}^{2}$ was found by fitting Eq. (2) to the thickness dependence of the effective anisotropy field, which was determined from the hard axis PMOKE hysteresis loops, dominated by a coherent magnetization rotation, for $d>2.3 \mathrm{~nm}$. The found value of $K_{s}$ is close to the one reported in Ref. 24 . 


\section{B. Ultrafast manipulation of magnetic anisotropy}

The magnetization precession was measured for several values of the external magnetic field (between 0.5 and $3.0 \mathrm{kOe}$ ) and Co layer thickness. To be sure that the sample was in a monodomain state and precession was homogeneous, the static hysteresis curves were measured in the same tilted field and the subsequent studies of the dynamics were confined to these fields being high enough to saturate the sample. Thus, the magnetization dynamics for the smallest thicknesses was measured with the strongest fields only. In order to derive the parameters that characterize the precession, such as amplitude $(A)$, frequency $(f)$, and damping $(\delta)$, the function

$$
m(t)=A \sin (2 \pi f t+\phi) e^{-\delta t}+B e^{-\gamma t}
$$

was fitted to the measured data. The last exponent corresponds to a nonmagnetic background, whereas the sine function with phase $\phi$ and exponential envelope represents an oscillation with damping. From here, the Gilbert damping constant was calculated as $\alpha=\delta /(2 \pi f)$.

The results of the measurements for a few selected values of the Co layer thickness and external magnetic field amplitude are displayed in Fig. 2 as black points. The plots show the pure oscillation with the nonmagnetic contribution subtracted. Vertical scales represent the change of magnetization normalized to the magnetization saturation value, i.e., expressed as Kerr rotation measured in dynamics divided by the one observed in statics in the saturation state. Both amplitude and frequency of the precession change distinctly with applied field amplitude
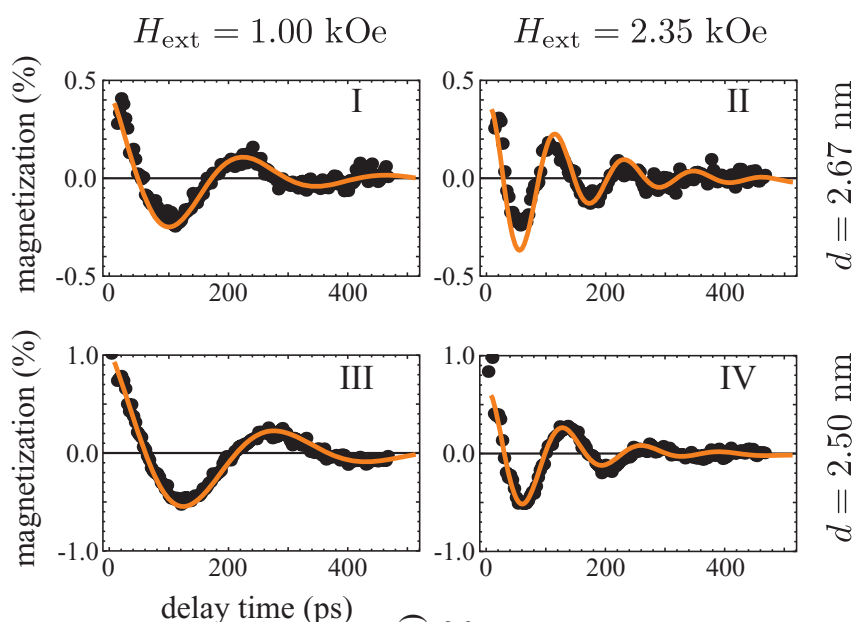

- measurement

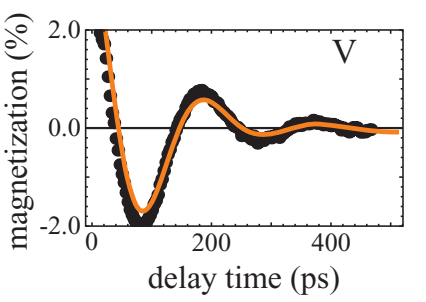

ฮี

FIG. 2. (Color online) Comparison of measured (black points) and calculated (orange lines) precession for selected thicknesses of the Co layer (rows) and magnetic field amplitude (columns). Units of vertical axes are expressed as percentage of magnetization saturation, i.e., measured Kerr rotation divided by the one observed in statics at saturation. and Co layer thickness, whereas the oscillation phase $\phi$ stays always the same.

The initial (before the pump pulse) magnetization orientation with the presence of the tilted external magnetic field was determined from the minimization of the magnetic anisotropy energy [Eq. (1)] in the $K_{\text {leff-only approximation with the }}$ Zeeman interaction $\left(-\vec{H}_{\text {ext }} \cdot \vec{M}\right)$ added. The orange points in Fig. 1 show the cosines of the initial magnetization orientation, for values of the Co layer thickness and the external field amplitude used in the experiment. Additionally, as a guide to the eye, the thickness dependencies for those field values are plotted as dashed orange lines. The horizontal line corresponds to the boundary case of an infinite field. For thicknesses above the SRT, the second anisotropy constant $\left(K_{2}\right)$ could eventually play a role. At the $-2 K_{2}<K_{1}<0$ region-according to Eq. (1) —an easy-cone state occurs, i.e., the magnetization prefers to be aligned at some angle with respect to the sample plane. However, in our case a tilted magnetic field itself is the factor that pulls the magnetization out of the plane, regardless of $K_{2}$. This means that from the point of view of our experiment, $K_{2}$ is insignificant, and the approximation with $K_{1 \text { eff }}$ only describes the observed effect well.

\section{Effective anisotropy model}

To describe the measured magnetization dynamics, a model involving the thermal changes of the effective anisotropy as a trigger for the precession behavior was developed. Magnetization precession with damping is governed by the Landau-Lifshitz-Gilbert (LLG) equation:

$$
\frac{d \vec{M}}{d t}=-\gamma\left(\vec{M} \times \vec{H}_{\mathrm{eff}}\right)+\frac{\alpha}{M}\left(\vec{M} \times \frac{d \vec{M}}{d t}\right) .
$$

$\vec{H}_{\text {eff }}$ denotes an effective magnetic field, which contains all magnetic fields affecting the sample. Here it consists of the external magnetic field applied to the sample and a total anisotropy field

$$
\vec{H}_{\text {eff }}=\vec{H}_{\text {ext }}+\vec{H}_{\text {tot }}
$$

\section{Anisotropy contribution}

To treat the total anisotropy field as a real field vector that affects the magnetization, within the approximation of the first anisotropy constant, it can be expressed as a vector perpendicular to the sample plane (along $\hat{z}$ axis), with a length depending on the actual magnetization orientation

$$
\vec{H}_{\text {tot }}=\hat{z} H_{1 \text { eff }} \cos \theta \text {. }
$$

For a positive $H_{1 \text { eff }}$ value, $\vec{H}_{\text {tot }}$ rotates the magnetization to the perpendicular direction, but in the case of a negative one, $\vec{H}_{\text {tot }}$ pulls the magnetization towards the plane instead.

The effective anisotropy field is assumed to change with temperature, as anisotropy constants and magnetization are temperature dependent. The temperature dependence of the magnetization was taken from interpolated experimental data from Ref. 25. The values of the magnetization saturation at zero temperature $M(0)=1.45 \times 10^{6} \mathrm{~A} / \mathrm{m}$ and the Curie temperature $T_{C}=1388 \mathrm{~K}$ were specified as thickness-independent (as the layer is thick enough ${ }^{26}$ ), and taken as for bulk 
cobalt. $^{27}$ The temperature dependence of the anisotropy in general was assumed to be proportional to a certain power of magnetization: ${ }^{12,13}$

$$
K(T) \propto M(T)^{n}
$$

The exponent $n$ depends on the order (i.e., the symmetry) of the constant. For the first order [Eq. (1)] $n=3$. This value would be applied to both $K_{v}$ and $K_{s}$ components, as they both are first-order constants. Nevertheless, some groups report that $K_{S}$ behaves differently. ${ }^{13,28-30}$ Keeping this in mind, in our work we have chosen the dependence with $n=3$ for $K_{v}$ only, whereas for $K_{s}$ the value of the exponent was determined from the fitting procedure.

\section{The role of temperature}

The major part of the incoming beam is reflected from the metallic surface; the remaining part is absorbed and heats the sample. However, because of the absorption, the deeper parts of the sample are affected by a lower energy of the beam. To get a depth-profile of energy absorption, at first we calculated the amplitude of the electromagnetic field of the beam (referred to as the incoming one) as a function of depth in the sample. We used a standard matrix method, ${ }^{20}$ considering reflection at the interfaces and absorption in the constituent layers (optical constants were taken from Ref. 31). On this basis we determined the fraction of the pulse energy that is absorbed in the sample at a certain depth.

The ultrafast heating of a spin system is often described with a three-temperature model ${ }^{5}$ (3TM), which assumes the existence of three reservoirs: electrons, spins, and a lattice. The baths are characterized by individual temperatures, $T_{e}, T_{s}$, and $T_{l}$, of electrons, spins, and lattice, respectively, that are also characterized by their individual heat capacities. These reservoirs are coupled to each other by specific coupling mechanisms and, generally speaking, react at different time scales. Electrons absorb energy of the pulse and next thermalize with the spins and the lattice ${ }^{32}$ within a few picoseconds. The anisotropy is supposed to depend mostly on the lattice temperature, while the demagnetizing field depends on the spin one. Exact solution of this problem with the help of a 3TM model requires the use of numerous parameters, such as individual temperatures and heat capacities for electrons, lattice, and spins and coupling parameters between respective reservoirs. Moreover, thermalization between baths in adjacent layers happens with different efficiencies. In order to simplify this model, we made the following assumptions. The only difference in considering either a $3 \mathrm{TM}$ or model with one temperature common for all baths (1TM), refers to the first few picoseconds, until the baths have thermalized. At longer time scales, calculation within both approaches should yield identical results. As we are interested in several (1-10) $\mathrm{GHz}$ dynamics, i.e., processes that happen on the time scale of several $(>50)$ picoseconds, processes of the time scale of a few $(<5)$ picoseconds are of less importance. Therefore, we assume that all baths at a certain depth in the sample are described by the same temperature and the heating occurs immediately after the light pulse and simultaneously in all metallic layers.

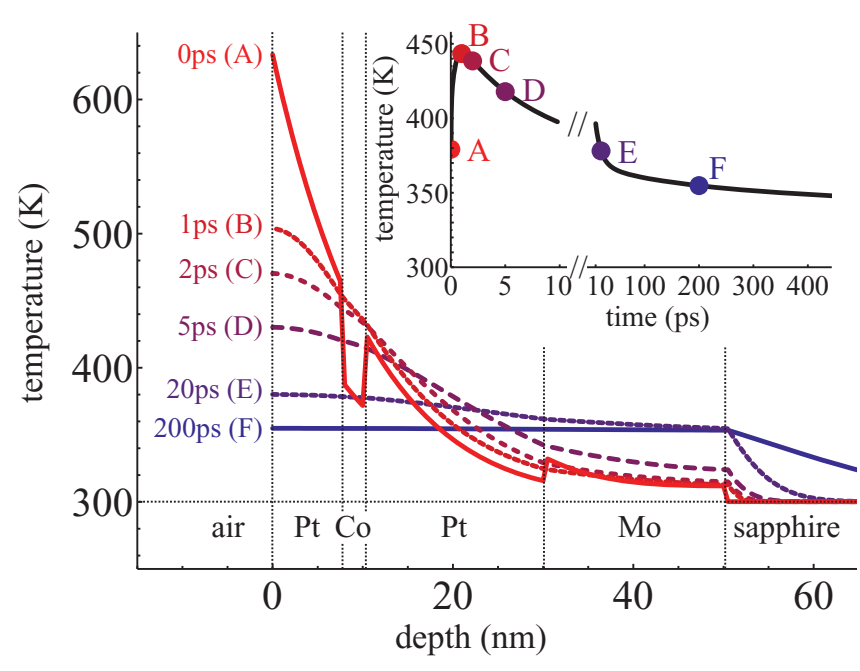

FIG. 3. (Color online) Calculated temperature profiles as a function of depth in the sample for selected time delays, for $d=2.5$ $\mathrm{nm}$. Discontinuities at the interfaces for zero time delay are due to different heat capacities of the constituent layers. Inset: temperature dynamics estimated for the middle of the Co layer; points marked as $\mathrm{A}-\mathrm{F}$ are taken from the relevant temperature profiles.

The initial temperature increase was converted from the calculated energy density using tables of standard enthalpy for bulk metals. ${ }^{33}$ Thermalization between layers was described by numerically solving the heat equation, with bulk values for thermal conductivities and heat capacities. ${ }^{34}$ The results of the calculations of the depth-dependent temperature profile are shown in Fig. 3, for selected time delays, for $d=2.5 \mathrm{~nm}$. Heat transfer along the perpendicular axis only was considered and the substrate was assumed as the main channel of energy dissipation. The planar size of the beam $(60 \mu \mathrm{m})$ was 3 orders of magnitude larger than the metallic layers thicknesses, so the planar heat transfer was neglected. As was also checked with the heat equation, this planar heat transfer happens on the microsecond time scale; i.e., the planar energy distribution was practically constant on the several-picosecond-time scale. From the calculated depth-profiles of temperature, the temperature dynamics in the Co layer itself was taken, as the one being important for magnetization dynamics.

\section{Excitation of precession}

In general the LLG equation is not applied to situations with changing value of magnetization saturation, ${ }^{17}$ which happens in our case. However, the calculated temperature increase in the Co layer is relatively small as compared to $T_{C}$. This means that the saturation magnetization does not change much with heating (less than $2 \%$ ), which makes the LLG equation eligible here. The anisotropy field, depending on a certain power of the magnetization, varies faster with temperature than the magnetization. A relatively small change of the effective magnetic field is sufficiently high to trigger the precession.

All discussed relations between parameters: the effective field, anisotropy constants, magnetization saturation, layer thickness, temperature, and time were involved in the LLG equation. All values of the physical quantities used in the calculations were taken either from textbooks ${ }^{27,34}$ (magnetization saturation at zero temperature, bulk anistropy 


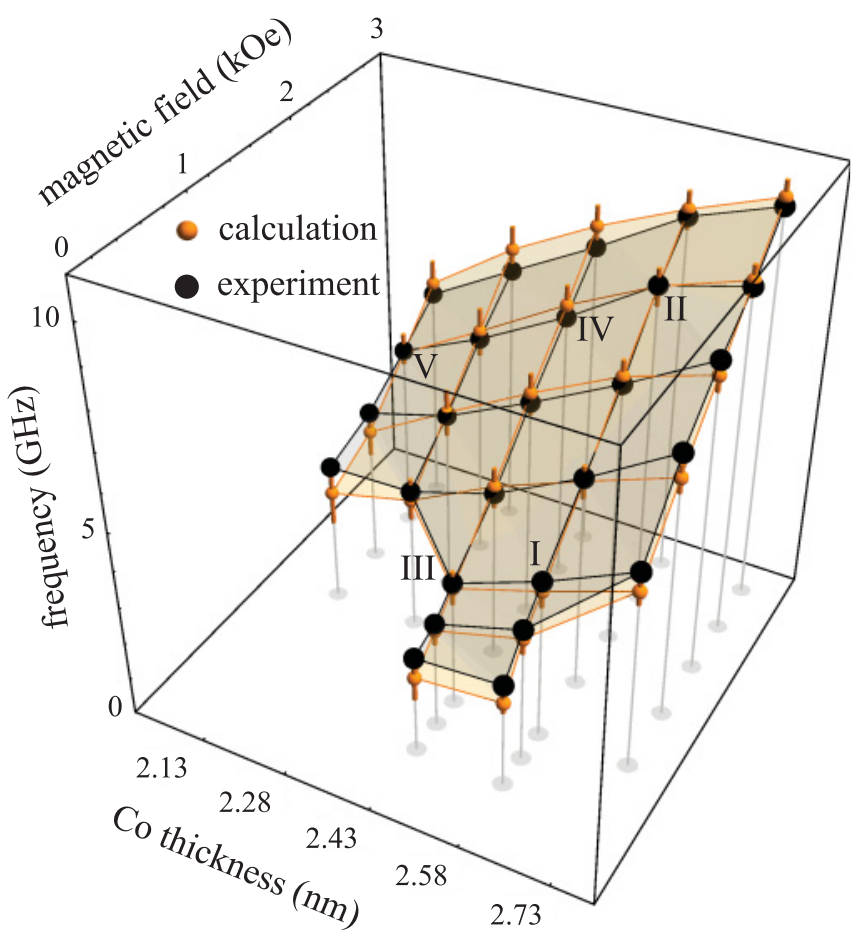

FIG. 4. (Color online) Frequency of measured (black) and calculated (orange) precession, as a function of Co layer thickness and magnetic field amplitude. Error bars: for the experimental points they are smaller than the size of the points; for the calculated points they are estimated from the errors of the particular experimental parameters involved in the calculations. Curves for points $\mathrm{I}-\mathrm{V}$ are plotted in Fig. 2.

constant, Curie temperature, thermodynamic quantities) or from the measurements (surface anisotropy constant from statics, Gilbert damping from dynamics). The exponent $n$ in the temperature dependence of the surface contribution to the anisotropy [Eq. (7)] was the only free parameter. The LLG equation was solved numerically, for all experimental values of the Co layer thickness and magnetic field amplitude-a set of precessional time-dependencies of the perpendicular component of the magnetization was obtained, to be compared to the experimental data. A single value of $n$ was chosen to make all calculated curves fit the measured data simultaneously. The value of that exponent was found to be $n=(3.2 \pm 0.4)$. It agrees well with the theoretical $n=3$, according to Callen and Callen. ${ }^{12}$ Comparison of the experimental curves (with non-magnetic contribution subtracted) with the calculation results obtained from the presented model for selected values of the Co layer thickness and magnetic field amplitude, with $n=3.2$, is shown in Fig. 2. All measured and calculated curves are represented by the derived frequencies and amplitudes of the precession in Figs. 4 and 5, respectively. Experimental values were determined quite precisely, their error bars are smaller than the size of the points. On the other hand, the error bars of calculated values are rather large, as many experimental parameters were involved in the calculations. Nevertheless, the general agreement is satisfactory.

It is worth noting that the amplitude is the only $n$-sensitive parameter and was used for the fitting procedure, while the frequency hardly changes with $n$. Analyzing the parameters

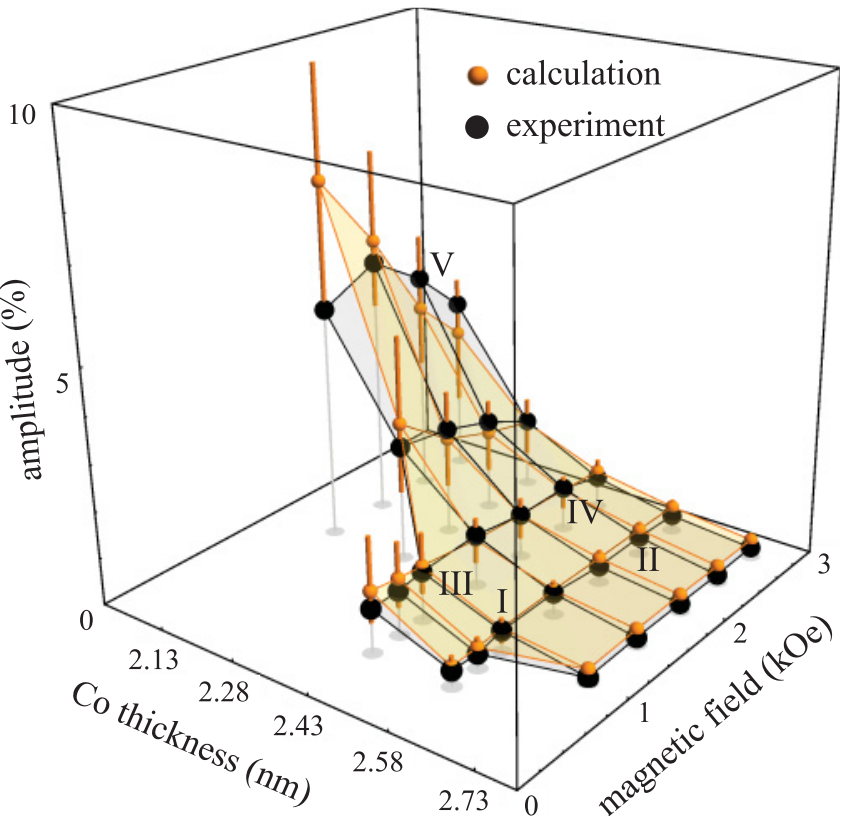

FIG. 5. (Color online) Amplitude of measured (black) and calculated (orange) precession, as a function of Co layer thickness and magnetic field amplitude. Units of amplitude are the percentage of magnetization saturation. Error bars: for the experimental points they are smaller than the size of the points; for the calculated points they are estimated from the errors of the particular experimental parameters involved in the calculations. Curves for points I-V are plotted in Fig. 2.

used in the model, we found that there are groups of parameters affecting either the amplitude or frequency more effectively. The amplitude of the precession depends on all the parameters playing a role in the dynamics: the temperature-dependencies of anisotropy field and magnetization saturation, the exponent in Eq. (7), the Curie temperature, and the incident energy intensity (which affects the initial temperature increase). Surprisingly, a negligible influence of the rate of temperature changes on the amplitude was found. On the other hand, the frequency is not sensitive to the above-mentioned quantities, whereas it is very sensitive to the initial (room-temperature) values of the anisotropy field.

The phase of the oscillations is the same for all curves, both in the experiments and in the calculations. The explanation of this fact lays in the tendency of changes of the effective anisotropy field. According to Eq. (2), positive contributions with $K_{v}$ and $K_{s}$ compete with the negative demagnetization term, but they decrease faster with temperature than the demagnetization [Eq. (7)]. A positive effective anisotropy field (easy axis case) decreases with heating, while a negative one (easy plane case) - increases its length (and becomes "more" negative). In both situations it results in the same tendency of changes of the effective magnetic field [Eq. (5)] with respect to the magnetization orientation. Thus, the magnetization precesses always in the same direction, which naturally results in the observed constant phase.

The Gilbert damping $\alpha$ was not a subject of theoretical consideration, but as mentioned earlier, the measured values were used for the calculations. Nevertheless, it did not affect 
the frequency and amplitude too much. It changes from about 0.1 at the highest thickness to 0.4 at the smallest one, in agreement with the tendency already reported before. ${ }^{18}$

\section{CONCLUSIONS}

Laser-induced magnetization precession in $\mathrm{Pt} / \mathrm{Co} / \mathrm{Pt}$ films was studied near the SRT as a function of the Co layer thickness and the external magnetic field amplitude. A significant decrease of frequency (twice) and increase of amplitude (an order of magnitude) was observed for reducing the Co layer thickness by $0.6 \mathrm{~nm}$ only. To describe the measured dependencies, we proposed a simplified model of laser-induced magnetization precession. We assumed that the mechanism that triggers precession in the sample is a thermal change of the perpendicular magnetic anisotropy, proportional to a certain power of magnetization and following thermal changes of the magnetization on a picosecond time scale. Within the model the measured dependence of the precession frequency was reproduced with satisfactory accuracy. Using the measured dependence of the precession amplitude we were also able to determine and confirm the theoretical value of the exponent of the temperature dependence of the surface contribution to the anisotropy.

Keeping in mind the mentioned sensitivity of the amplitude or frequency to various parameters, the model seems to be appropriate for the fitting procedure. Using the amplitude or frequency, depending on parameters that are known (or measured) and that are to be found, treating the other one as a reference, one can determine physical quantities that are hard to be studied locally. This provides an opportunity to probe, e.g., Curie temperature, anisotropy, or thermodynamic parameters with micrometer resolution, which can be especially interesting for low-dimensional objects such as patterned systems and nanostructures.

\section{ACKNOWLEDGMENTS}

The authors thank A. F. van Ettger, A. J. Toonen, and A. van Roij for technical support, A. R. Khorsand for help with the experimental setup and stimulating discussions, and Z. Kurant for help with sample characterization. The work was sponsored by FANTOMAS (Project EC FP7, Grant No. 214810). *jankis@uwb.edu.pl

${ }^{1}$ A. Kirilyuk, A. V. Kimel, and T. Rasing, Rev. Mod. Phys. 82, 2731 (2010).

${ }^{2}$ A. Taratorin, S. Yuan, and V. Nikitin, J. Appl. Phys. 93, 6444 (2003).

${ }^{3}$ C. D. Stanciu, Ph.D. thesis, Radboud University Nijmegen, 2008.

${ }^{4}$ L. Guidoni, E. Beaurepaire, and J.-Y. Bigot, Phys. Rev. Lett. 89, 017401 (2002).

${ }^{5}$ E. Beaurepaire, J.-C. Merle, A. Daunois, and J.-Y. Bigot, Phys. Rev. Lett. 76, 4250 (1996).

${ }^{6}$ A. V. Kimel, A. Kirilyuk, P. A. Usachev, R. V. Pisarev, A. M. Balbashov, and T. Rasing, Nature (London) 435, 655 (2005).

${ }^{7}$ C. D. Stanciu, F. Hansteen, A. V. Kimel, A. Kirilyuk, A. Tsukamoto, A. Itoh, and T. Rasing, Phys. Rev. Lett. 99, 047601 (2007).

${ }^{8}$ G. Ju, A. Vertikov, A. V. Nurmikko, C. Canady, G. Xiao, R. F. C. Farrow, and A. Cebollada, Phys. Rev. B 57, R700 (1998).

${ }^{9}$ M. van Kampen, C. Jozsa, J. T. Kohlhepp, P. LeClair, L. Lagae, W. J. M. de Jonge, and B. Koopmans, Phys. Rev. Lett. 88, 227201 (2002).

${ }^{10}$ F. Hansteen, A. Kimel, A. Kirilyuk, and T. Rasing, Phys. Rev. Lett. 95, 047402 (2005).

${ }^{11}$ F. Hansteen, A. Kimel, A. Kirilyuk, and T. Rasing, Phys. Rev. B 73, 014421 (2006).

${ }^{12}$ E. Callen and H. Callen, Phys. Rev. B 139, A455 (1965).

${ }^{13}$ M. Farle, Rep. Prog. Phys. 61, 755 (1998).

${ }^{14}$ J. B. Staunton, L. Szunyogh, A. Buruzs, B. L. Gyorffy, S. Ostanin, and L. Udvardi, Phys. Rev. B 74, 144411 (2006).

${ }^{15}$ K. Zakeri, T. Kebe, J. Lindner, and M. Farle, Phys. Rev. B 73, 052405 (2006).

${ }^{16}$ W. J. M. de Jonge, P. J. H. Bloemen, and F. J. A. den Broeder, Ultrathin Magnetic Structures (Springer-Verlag, Berlin, 1994).

${ }^{17}$ J.-Y. Bigot, M. Vomir, L. H. F. Andrade, and E. Beaurepaire, Chem. Phys. 318, 137 (2005).
${ }^{18}$ S. Mizukami, E. P. Sajitha, D. Watanabe, F. Wu, T. Miyazaki, H. Naganuma, M. Oogane, and Y. Ando, Appl. Phys. Lett. 96, 152502 (2010)

${ }^{19}$ M. A. C. de Araújo, R. Silva, E. de Lima, D. P. Pereira, and P. C. de Oliveira, Appl. Opt. 48, 393 (2009).

${ }^{20}$ Š. Višňovský, Optics in Magnetic Multilayers and Nanostructures (CRC Press/Taylor \& Francis, London, 2006).

${ }^{21}$ M. Kisielewski, A. Maziewski, M. Tekielak, A. Wawro, and L. T. Baczewski, Phys. Rev. Lett. 89, 087203 (2002).

${ }^{22}$ L. Néel, J. Phys. Radiat. 15, 225 (1954).

${ }^{23}$ R. Allenspach, M. Stampanoni, and A. Bischof, Phys. Rev. Lett. 65, 3344 (1990).

${ }^{24}$ N. W. E. McGee, M. T. Johnson, J. J. De Vries, and J. Aan De Stegge, J. Appl. Phys. 73, 3418 (1993).

${ }^{25}$ M. D. Kuz'min, Phys. Rev. Lett. 94, 107204 (2005).

${ }^{26}$ R. Rausch and W. Nolting, J. Phys.: Condens. Matter 21, 376002 (2009).

${ }^{27}$ J. Stöhr and H. C. Siegmann, Magnetism: From Fundamentals to Nanoscale Dynamics (Springer-Verlag, Berlin, 2006).

${ }^{28}$ O. Fruchart, J.-P. Nozieres, and D. Givord, J. Magn. Magn. Mater. 165, 508 (1997).

${ }^{29} \mathrm{P}$. Jensen and K. Bennemann, Solid State Commun. 100, 585 (1996).

${ }^{30}$ A. Hucht and K. D. Usadel, Phys. Rev. B 55, 12309 (1997).

${ }^{31}$ E. D. Palik, ed., Handbook of Optical Constants of Solids (Academic Press/Elsevier, New York, 1998).

${ }^{32}$ B. Koopmans, G. Malinowski, F. Dalla Longa, D. Steiauf, M. Fähnle, T. Roth, M. Cinchetti, and M. Aeschlimann, Nat. Mater. 9, 259 (2010).

${ }^{33}$ NIST-JANAF Thermochemical Tables, [http://kinetics.nist.gov/ janaf/] and Research Information Database, [http://riodb.ibase. aist.go.jp/].

${ }^{34}$ CRC Handbook of Chemistry and Physics, 88th ed. (CRC Press, Boca Raton, 2007). 